\title{
Academic Research Enhancement Awards (Area)
}

National Cancer Institute

\section{Source}

National Cancer Institute. Academic Research Enhancement Awards (Area). NCI

Thesaurus. Code C18635.

The three goals of the AREA program are: to support meritorious research, to strengthen the research environment of the institution, and to expose students to research. The focus is not on course work but on hands-on meritorious research. 